\title{
Anthocyanin-rich Aronia melanocarpa extract improves body temperature maintenance in healthy women with a cold constitution
}

\author{
Keisuke Sonoda ${ }^{2}$, Wataru Aoi ${ }^{*}$, Tomoaki Iwata ${ }^{2}$ and Yanmei Li ${ }^{3}$
}

\begin{abstract}
Purpose: Specific anthocyanin-rich dietary factors have been shown to improve metabolic functions associated with thermogenesis in animal studies. Aronia melanocarpa, commonly known as wild chokeberry, contains a high level of anthocyanin that would be expected to maintain body temperature through thermogenesis. We here investigated the effects of Aronia melanocarpa extracts on body temperature and peripheral blood flow in healthy women with a cold constitution.
\end{abstract}

Methods: A pre/post comparison trial was performed in 11 women with a cold constitution, who were taking Aronia melanocarpa extracts (150 mg/day) for 4 weeks. Physiological and biochemical parameters, along with psychological tests were examined.

Results: The subjects' body surface temperature was significantly higher in the post-trial than in the pre-trial. In psychological tests, factors related to cold were significantly improved by Aronia intake. On the other hand, peripheral blood flow was not affected by Aronia supplementation. Plasma noradrenalin level was significantly elevated by Aronia intake, and subjects with a higher level of 8-hydroxy-2'-deoxyguanosine in the pre-trial showed decreased levels in the post-trial.

Conclusions: These data suggest that dietary Aronia melanocarpa extract improves the maintenance of body temperature in healthy women with a cold constitution, which may be mediated by noradrenalin and oxidative stress levels.

Keywords: Anthocyanin; Thermogenesis; Body surface temperature; Blood flow; Cold feeling

\section{Background}

Peripheral cold constitution is a popular symptom among women and causes various health disorders such as shoulder stiffness, headache, swelling, sleeplessness, frequent urination, peripheral numbness, limb pain, chilblains, and purple fingernails (Melby 2007; Masuda et al. 2011). Body temperature is maintained by thermogenesis and thermal radiation. Thus, a reduction in thermogenesis leads to a cold feeling. In addition, it is thought that disturbances in peripheral circulation, induced by the contraction of peripheral vessels, play a role in thermal dysregulation. The contraction of peripheral vessels can be caused by

\footnotetext{
* Correspondence: waoi@kpu.ac.jp

'Laboratory of Health Science, Graduate School of Life and Environmental Sciences, Kyoto Prefectural University, 1-5 Hangi-cho Shimogamo, Sakyo-ku, Kyoto 606-8522, Japan

Full list of author information is available at the end of the article
}

sympathetic nerve activation, a decrease in endotheliumderived relaxant factor, or an increase in vasoconstrictor levels (Matz et al. 2000; Shepherd 1990). Therefore, if thermogenesis and peripheral circulation can be improved, the cold feeling may be alleviated.

Anthocyanins are the largest group of water-soluble pigments in the plant kingdom and are abundant in fruits, vegetables, beans, and red wine. Dietary anthocyanins have been shown to play an important role in the prevention of life-related common diseases, mainly as a result of their strong antioxidant and anti-inflammatory effects (Desjardins et al. 2012; Cuevas-Rodríguez et al. 2010). Anthocyanins improve the peripheral circulatory system by lowering blood pressure, maintaining the proper permeability and elasticity of arterial vessels, and activate nitric oxide $(\mathrm{NO})$ to promote endothelial vasodilation 
(Jennings et al. 2012; Ojeda et al. 2010; Côrtes et al. 2002). In addition, several animal studies have shown that specific anthocyanins improve metabolism-associated thermogenesis in peripheral organs (DeFuria et al. 2009; Takikawa et al. 2010). Therefore, such dietary factors may be effective in improving cold constitution in humans.

Fruits berries are a dietary source of anthocyanin as well as many other essential nutritional components. Aronia melanocarpa is especially rich in polyphenols, in particular, anthocyanins as well as procyanidins and flavonoids (Kokotkiewicz et al. 2010). Previously, it has been shown that the intake of Aronia extract improves vascular function and metabolic activity along with antioxidant and anti-inflammatory (Broncel et al. 2010; Qin et al. 2012; Zapolska-Downar et al. 2012), which may contribute to the improvement of thermogenesis and peripheral circulation. Therefore, the aim of our study was to investigate the effects of Aronia melanocarpa extract supplementation on peripheral body temperature and blood flow in healthy women with a cold constitution.

\section{Results}

Body surface temperature and peripheral blood flow

The maximum value for body temperature after $20 \mathrm{~min}$ of acclimatization in an air-conditioned room was significantly higher in the post-trial compared with the pre-trial measurement $(\mathrm{P}=0.004)$ (Figure $1 \mathrm{~A})$. The relative mean value of blood flow in the subjects' hands was calculated. The values were significantly decreased by cold water immersion over time in both the pre- and post-trials (Figure 1B). It showed a tendency towards suppression by 4 weeks of Aronia supplementation, but not significantly affected.

\section{Blood and urine parameters}

The concentration of plasma noradrenalin significantly increased in the post-trial compared with the pre-trial $(P=0.027)$ (Table 1$)$. In contrast, no significant changes were detected in plasma adrenalin, dopamine, nitrite/nitrate or cyclic guanosine monophosphate (cGMP) concentrations between pre- and post-trials. No significant change was observed in urine 8-hydroxydeoxyguanosine (8OHdG) levels between trials, although it was significantly decreased from $10.7 \pm 3.0 \mathrm{ng} / \mathrm{mL}$ to $6.7 \pm 3.4 \mathrm{ng} / \mathrm{mL}$ by Aronia intake in 5 subjects who had baseline $8 \mathrm{OHdG}$ levels above $5 \mathrm{ng} / \mathrm{mL}$ $(\mathrm{P}=0.022)$ (Figure 2).

\section{Psychological tests}

The value related to a cold feeling in the hand, foot, and hip was significantly improved after supplementation compared with the baseline (Table 2). On the other hand, we did not observe any significant changes in the value for a cold feeling of the back and shoulder stiffness between trials.

\section{Discussion}

The present study revealed the following novel observations: (1) Aronia supplementation for 4 weeks elevated body surface temperature, (2) Aronia increased plasma noradrenalin concentration, and (3) Aronia attenuated the cold feeling in all 4 limbs and the hip, as determined by the VAS test, in 11 women with a cold constitution. Cold constitution causes various symptoms such as shoulder stiffness, headache, swelling, frequent urination, peripheral numbness, lumbar pain, and limb pain. Thus, the attenuation of the cold feeling in all 4 limbs and the hip by Aronia supplementation is associated with an improvement in cold constitution. Previously, it has been shown that several polyphenols can activate thermogenesis by improving the metabolism in metabolic organs and cells in animal and cell culture studies (Alberdi et al. 2013; Dulloo et al. 2000; Moon et al. 2013; Shixian et al. 2006); however, it has not been shown in humans. To the best of our knowledge, our observations are the first to demonstrate that polyphenol-rich food extracts improve a cold constitution in human subjects through thermoregulation and redox regulation.
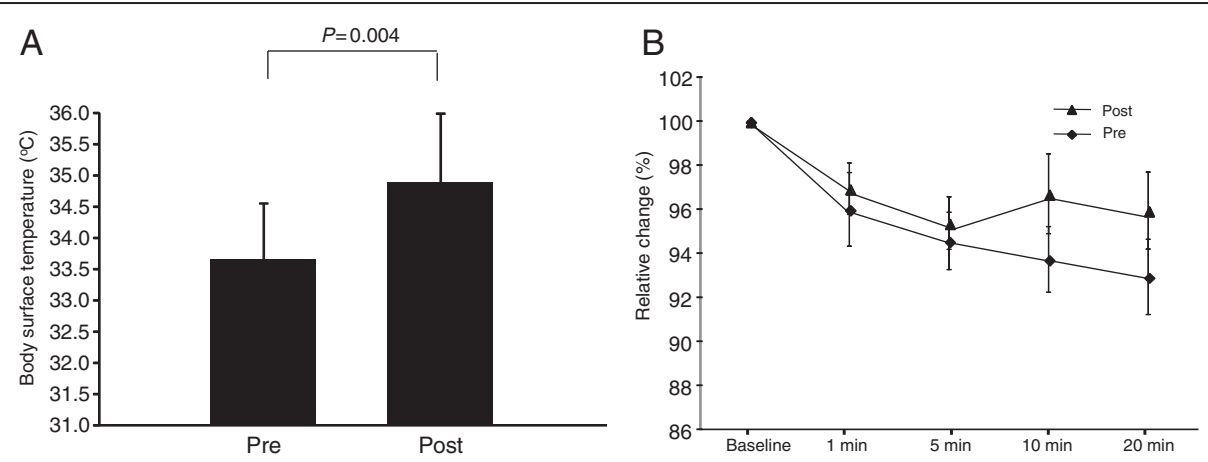

Figure 1 Body surface temperature and peripheral blood flow. (A) The maximum value for body temperature after 20 min of acclimatization in an air-conditioned room was measured. (B) The blood flow was examined during immersing their right hand into cold water for 20 min in a time-course manner. Values represent the mean \pm standard deviation obtained from 11 subjects. Pre, pre trial; Post, post trial. 
Table 1 Concentration of plasma and urine parameters

\begin{tabular}{lcc}
\hline & Pre & Post \\
\hline Plasma & & \\
Adrenalin $\left(\mathrm{ng} \cdot \mathrm{mL}^{-1}\right)$ & $0.025 \pm 0.014$ & $0.031 \pm 0.018$ \\
Noradrenalin $\left(\mathrm{ng} \cdot \mathrm{mL}^{-1}\right)$ & $0.225 \pm 0.070$ & $0.298 \pm 0.0999^{\mathrm{a}}$ \\
Dopamine $\left(\mathrm{ng} \cdot \mathrm{mL}^{-1}\right)$ & $0.013 \pm 0.009$ & $0.013 \pm 0.005$ \\
Cyclic GMP $\left(\mathrm{ng} \cdot \mathrm{mL}^{-1}\right)$ & $3.14 \pm 1.04$ & $3.24 \pm 1.10$ \\
$\quad$ Nitrite $\left(\mu \mathrm{mol} \cdot \mathrm{L}^{-1}\right)$ & $1.00 \pm 0.00$ & $1.00 \pm 0.00$ \\
$\quad$ Nitrate $\left(\mu \mathrm{mol} \cdot \mathrm{L}^{-1}\right)$ & $23.4 \pm 8.1$ & $27.2 \pm 12.7$ \\
Urine & & \\
8OHdg $\left(\mathrm{ng} \mathrm{mL} \mathrm{mL}^{-1}\right)$ & $6.25 \pm 4.74$ & $6.50 \pm 3.70$ \\
\hline
\end{tabular}

The plasma levels of catecholamines, cyclic GMP, and nitrite/nitrate as well as the urinary level of $8 \mathrm{OHdG}$ were examined. Pre, pre-trial; Post, post-trial.

a, significant deference between the Pre at the level of $P<0.05$.

Aronia melanocarpa contains polyphenols such as anthocyanins, procyanidins, and flavonoids that can improve nutrient metabolism and possess antioxidant and anti-inflammatory properties. Previously, it has been shown that some factors elevate thermogenesis in peripheral metabolic organs. Takikawa et al. (2010) reported that an anthocyanin, cyanidin 3-glucoside, enhances adiponectin secretion and upregulates the expression of thermogenesis-related proteins, such as uncoupling proteins, in isolated rat adipocytes. Cyanidin 3-glucoside also activates adenosine monophosphate (AMP)-activated protein kinase (AMPK) in skeletal muscle and liver. In addition, quercetin-rich onion peel extract elevates uncoupling protein-1 expression and inhibits adipogenesis in both 3 T3-L1 cells and in rats fed high-fat diets (Moon et al. 2013). In addition to the direct effect of anthocyanins on improving metabolism in metabolic organs, circulating levels of catecholamine may be an inducer of thermogenesis in these organs. It is well

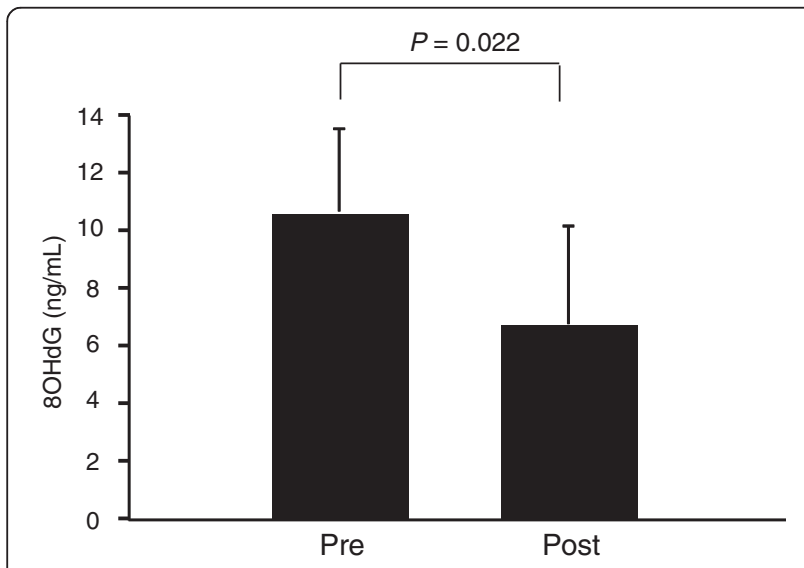

Figure 2 Urine $80 \mathrm{HdG}$. Urine $80 \mathrm{HdG}$ levels were measured in subjects who had baseline $80 \mathrm{HdG}$ levels above $5 \mathrm{ng} / \mathrm{mL}$. Values represent the mean \pm standard deviation obtained from 5 subjects. Pre, pre trial; Post, post trial.
Table 2 Psychological test for the examination of subjective peripheral blood flow

\begin{tabular}{lcc}
\hline & Pre & Post \\
\hline Cold feeling & & \\
Hand and foot & $3.18 \pm 1.54$ & $4.17 \pm 0.84^{\mathrm{a}}$ \\
Hip & $3.18 \pm 1.54$ & $4.23 \pm 0.82^{\mathrm{a}}$ \\
Back & $3.64 \pm 1.21$ & $4.26 \pm 0.77$ \\
Shoulder stiffness & $2.91 \pm 1.45$ & $3.43 \pm 0.97$ \\
\hline
\end{tabular}

The subjects' state of psychological stress was assessed using a questionnaire regarding cold feelings in the hand/foot, hip, and back as well as shoulder stiffness. The questionnaire consisted of 5 responses, namely, 1 , feel very cold or stiffness; 2, feel somewhat cold or stiffness; 3 , do not feel anything; 4 , almost do not feel cold or stiffness at all. Values represent the mean \pm standard the deviation obtained from 11 subjects. Pre, pre-trial; Post, post-trial. ${ }^{a}$, significant difference from the Pre at the level of $P<0.05$.

known that noradrenaline elevates thermogenesis via cyclic AMP and uncoupling proteins after binding to the beta-adrenalic receptor in adipose and skeletal muscle tissues (Lowell et al. 2000; Rehnmark et al. 1990). We found increased levels of plasma noradrenalin after 4 weeks of Aronia intake, which may contribute to the attenuation of cold feeling through thermogenesis. On the other hand, autonomic nerve regulation may be associated with elevated noradrenalin, although it remains unclear. AMPK can be activated by some anthocyanins and is closely associated with sympathetic nervous system regulation. Several studies have shown that central AMPK stimulates sympathetic nerve activity that can induce the secretion of noradrenalin (Tanida et al. 2011; Viollet et al. 2003).

Reactive oxygen species generated in the vascular endothelium can then react with NO, reducing its vasoactive levels (Förstermann 2010). This diminishes the response to endothelium-dependent vasodilators via the formation of peroxynitrite anions $\left(\mathrm{ONOO}^{-}\right)$, a highly reactive intermediate with strong cytotoxic potency. Thus, antioxidant factors can promote a vasodilator effect of NO by scavenging reactive oxygen species. In fact, the intake of dietary antioxidants including vitamins, carotenoids, and polyphenols has been reported to show vasodilation action (Barona et al. 2012; Pleiner et al. 2008; Zhao et al. 2011). A number of studies have shown that flavonoids are superior to vitamins $\mathrm{E}$ and $\mathrm{C}$ as antioxidant agents. In addition, certain polyphenols have been observed to reduce the generation of reactive oxygen species from the endothelium or phagocytes (Cai et al. 1997), which can also contribute to decreasing the oxidative stress level. Aronia has been shown to attenuate exercise-induced oxidative damage associated with an improvement in exercise tolerance and other physiological parameters (Pilaczynska-Szczesniak et al. 2005). In the present study, dietary Aronia extracts caused a reduction in urine $8 \mathrm{OHdG}$ levels in subjects with a higher oxidative stress level before the trial. This may result in endothelium vasodilation by reducing oxidative stress. On the other hand, noradrenalin 
functions as an endothelium vasoconstrictor in the peripheral vein. Thus, the increase of noradrenalin by Aronia intake may lead to contraction of the peripheral vein. Nevertheless, blood flow was not decreased by Aronia intake, which suggests that vasodilation induced by reducing oxidative stress may compensate for the vasocontraction in response to noradrenalin, leading to an improvement in the cold feeling.

\section{Conclusion}

Body surface temperature and plasma noradrenalin levels were significantly elevated by Aronia supplementation. In psychological tests, factors related to cold were significantly decreased by Aronia intake. Subjects with higher 8OHdG levels at baseline showed decreased levels in the post-trial period. These data suggest that dietary Aronia melanocarpa extracts improve the maintenance of body temperature in healthy women with a cold constitution, which may be mediated by noradrenalin and oxidative stress levels. On the basis of the results of this pilot study, further research is required in a placebo-controlled study to determine whether these findings can be generalized to a larger population.

\section{Methods}

\section{Subjects}

Twelve healthy Japanese women, aware of their cold constitution, were recruited for this study using the following inclusion criteria: 40-60 years of age, normal menstrual cycle and dietary habits, and no severe physical or psychological illnesses. A pre-examination was performed to objectively determine the degree of the women's cold constitution. Subjects with severe cold constitution were screened by performing a pre-test where subjects immersed their right hand into cold water for $20 \mathrm{~min}$ and subsequently, the blood flow was determined. Ultimately, 11 women $(33.2 \pm 4.9 \mathrm{yr})$ who were judged to have high cold constitution were asked to participate in this study and underwent a medical examination. The examination included an interview with a physician and blood and urine analyses before commencement of the study. All subjects were free of signs, symptoms, and history of any overt chronic disease. This study was approved by the ethics committee of Shiba Palace Clinic and all subjects signed a consent form after reading the study protocol.

\section{Aronia tablet}

Frozen Aronia melanocarps fruits were extracted by ethanol/water, followed by chromatography and spray drying. The whole process was performed under low temperature and with nitrogen protection, so that the anthocyanins may not be oxidized during the purification. Aronia tablets were comprised after added calcium stearate and silicon dioxide. Each tablet $(250 \mathrm{mg})$ included Aronia melanocarpa extract (50 mg), crystalline cellulose (25 mg), and starch hydrolysate $(162.5 \mathrm{mg})$, calcium stearate (7.5 mg), and silicon dioxide $(5 \mathrm{mg})$. The content of anthocyanin contained in Aronia melanocarpa extract was equivalent to $35 \% \mathrm{w} / \mathrm{w}$.

\section{Study design}

A pre/post comparison trial was performed. Body surface temperature, peripheral blood flow, blood and urine biochemical parameters, and psychological tests related to cold were measured for baseline measurements. All subjects took 3 Aronia tablets, daily, with water $30 \mathrm{~min}$ after breakfast for 4 weeks. The measurements were taken the day after the final tablet ingestion. Subjects were asked to refrain from consuming caffeine and alcohol, and intense physical activity $24 \mathrm{~h}$ before each trial.

\section{Body surface temperature and peripheral blood flow}

Peripheral body temperature was analyzed by thermography (Neo Thermo TVS-700, Nippon Avionics Co., Ltd., Tokyo, Japan). Subjects were acclimatized to an air-conditioned (25 $\pm 11^{\circ} \mathrm{C} / 50 \pm 10 \%$ room humidity) room for $20 \mathrm{~min}$ and the temperature of their right hand was measured using thermography. The point of maximum temperature in the thermogram image was examined.

Blood flow on the tip of the middle finger was measured using a laser Doppler flowmeter (PMEGAZONE OZ-1, OMEGAWAVE Ltd, Tokyo, Japan). Subjects immersed their right hand into cold water for $20 \mathrm{~min}$ and the blood flow was examined in a time-course manner.

\section{Blood and urine parameters}

Subjects provided a urine specimen from an overnight accumulation, returned to the laboratory at 9:00 am while maintaining their fast, sat on a chair, and were made to rest. Subsequently, blood was collected from the antecubital vein. Immediately after collection, each blood sample was centrifuged and plasma was obtained. The plasma levels of catecholamines (adrenalin, noradrenalin, and dopamine) and nitrite/nitrate were measured using high performance liquid chromatography. Plasma cGMP levels were measured by radioimmunoassay (YAMASA Co., Ltd., Chiba, Japan). The urine concentration of $8 \mathrm{OHdG}$, a marker of deoxyribonucleic acid oxidative damage, was measured using an enzyme-linked immunsorbent assay kit (Japan Institute for the Control of Ageing, Fukuroi City, Shizuoka, Japan).

\section{Psychological tests}

The subjects' state of psychological stress was assessed using a questionnaire. The questionnaire consisted of 5 responses, namely, 1, feel very cold; 2, feel somewhat cold; 3, do not feel anything; 4, almost do not feel cold; 5 , do not feel cold at all, for questions regarding cold feelings in the hand/foot, hip, and back. The subjects 
selected their perception of the current state of their hand, foot, hip, and back from the 5 sections. Similarly, a questionnaire was used to examine the degree of shoulder stiffness.

\section{Statistical analyses}

The differences between pre- and post-experiment parameters were evaluated by the Wilcoxon signed-rank test. Statistical significance was accepted at $\mathrm{P}<0.05$. All data are reported as the mean \pm standard deviation.

\section{Competing interests}

The authors declare that they have no competing interests.

\section{Authors' contributions}

$\mathrm{KS}, \mathrm{Tl}$, and $\mathrm{YL}$ performed design of research and experiments; KS and WA analyzed data; KS, WA, TI and YL interpreted results of experiments; WA drafted the manuscript. All authors read and approved the final manuscript.

\section{Author details}

'Laboratory of Health Science, Graduate School of Life and Environmental Sciences, Kyoto Prefectural University, 1-5 Hangi-cho Shimogamo, Sakyo-ku, Kyoto 606-8522, Japan. ${ }^{2}$ BGG Japan Co., Ltd, 8F YN Ginza Building, 8-14-11 Tokyo, Chuo-ku, Tokyo, Japan. ${ }^{3}$ Beijing Gingko Group Co., Ltd, 3F-KeHaiFulin Building, The Chinese Academy of Agricultural Sciences, No.12, Zhongguancun South Avenue, Beijing, Haidian District, China.

Received: 23 August 2013 Accepted: 13 November 2013 Published: 21 November 2013

\section{References}

Alberdi G, Rodríquez VM, Miranda J, Macarulla MT, Churruca I, Portillo MP (2013) Thermogenesis is involved in the body-fat lowering effects of resveratrol in rats. Food Chem 141:1530-1535

Barona J, Aristizabal JC, Blesso CN, Volek JS, Fernandez ML (2012) Grape polyphenols reduce blood pressure and increase flow-mediated vasodilation in men with metabolic syndrome. J Nutr 142:1626-1632

Broncel M, Kozirog M, Duchnowicz P, Koter-Michalak M, Sikora J, Chojnowska-Jezierska J (2010) Aronia melanocarpa extract reduces blood pressure, serum endothelin, lipid, and oxidative stress marker levels in patients with metabolic syndrome. Med Sci Monit 16:CR28-CR34

Cai Q, Rahn RO, Zhang R (1997) Dietary flavonoids, quercetin, luteolin and genistein, reduce oxidative DNA damage and lipid peroxidation and quench free radicals. Cancer Lett 119:99-107

Côrtes SF, Valadares YM, de Oliveira AB, Lemos VS, Barbosa MP, Braga FC (2002) Mechanism of endothelium-dependent vasodilation induced by a proanthocyanidin-rich fraction from Ouratea semiserrata. Planta Med 68:412-415

Cuevas-Rodríguez EO, Dia VP, Yousef GG, García-Saucedo PA, López-Medina J, Paredes-López O, Gonzalez de Mejia E, Lila MA (2010) Inhibition of pro-inflammatory responses and antioxidant capacity of Mexican blackberry (Rubus spp.) extracts. J Agric Food Chem 58:9542-9548

DeFuria J, Bennett G, Strissel K, Perfield JW, 2nd, Milbury PE, Greenberg AS, Obin MS (2009) Dietary blueberry attenuates whole-body insulin resistance in high fat-fed mice by reducing adipocyte death and its inflammatory sequelae. J Nutr 139:1510-1516

Desjardins J, Tanabe S, Bergeron C, Gafner S, Grenier D (2012) Anthocyanin-rich black currant extract and cyanidin-3-O-glucoside have cytoprotective and anti-inflammatory properties. J Med Food 15:1045-1050

Dulloo AG, Seydoux J, Girardier L, Chantre P, Vandermander J (2000) Green tea and thermogenesis: interactions between catechin-polyphenols, caffeine and sympathetic activity. Int J Obes Relat Metab Disord 24:252-258

Förstermann U (2010) Nitric oxide and oxidative stress in vascular disease. Pflugers Arch 459:923-939

Jennings A, Welch AA, Fairweather-Tait SJ, Kay C, Minihane AM, Chowienczyk P. Jiang B, Cecelja M, Spector T, Macgregor A, Cassidy A (2012) Higher anthocyanin intake is associated with lower arterial stiffness and central blood pressure in women. Am J Clin Nutr 96:781-788
Kokotkiewicz A, Jaremicz Z, Luczkiewicz M (2010) Aronia plants: a review of traditional use, biological activities, and perspectives for modern medicine. J Med Food 13:255-269

Lowell BB, Spiegelman BM (2000) Towards a molecular understanding of adaptive thermogenesis. Nature 404:652-660

Masuda H, Mori N, Hirooka S, Matsui Y, Tsukiyama M, Watanabe Y, Nadamoto T (2011) Effects of winter savory (satureja montana I.) on peripheral body temperature of people who experience a 'Feeling of Cold' (Hie-Sho). Food Sci Technol Res 17:429-436

Matz RL, Schott C, Stoclet JC, Andriantsitohaina R (2000) Age-related endothelial dysfunction with respect to nitric oxide, endothelium-derived hyperpolarizing factor and cyclooxygenase products. Physiol Res 49:11-18

Melby MK (2007) Chilliness: a vasomotor symptom in Japan. Menopause 14:752-759

Moon J, Do HJ, Kim OY, Shin MJ (2013) Antiobesity effects of quercetin-rich onion peel extract on the differentiation of 3 T3-L1 preadipocytes and the adipogenesis in high fat-fed rats. Food Chem Toxicol 58C:347-354

Ojeda D, Jiménez-Ferrer E, Zamilpa A, Herrera-Arellano A, Tortoriello J, Alvarez L (2010) Inhibition of angiotensin convertin enzyme (ACE) activity by the anthocyanins delphinidin- and cyanidin-3-O-sambubiosides from Hibiscus sabdariffa. J Ethnopharmacol 127:7-10

Pilaczynska-Szczesniak L, Skarpanska-Steinborn A, Deskur E, Basta P, Horoszkiewicz-Hassan M (2005) The influence of chokeberry juice supplementation on the reduction of oxidative stress resulting from an incremental rowing ergometer exercise. Int I Sport Nutr Exerc Metab 15:48-58

Pleiner J, Schaller G, Mittermayer F, Marsik C, MacAllister RJ, Kapiotis S, Ziegler S, Ferlitsch A, Wolzt M (2008) Intra-arterial vitamin C prevents endothelial dysfunction caused by ischemia-reperfusion. Atherosclerosis 197:383-391

Qin B, Anderson RA (2012) An extract of chokeberry attenuates weight gain and modulates insulin, adipogenic and inflammatory signalling pathways in epididymal adipose tissue of rats fed a fructose-rich diet. Br J Nutr 108:581-587

Rehnmark S, Néchad M, Herron D, Cannon B, Nedergaard J (1990) Alpha- and beta-adrenergic induction of the expression of the uncoupling protein thermogenin in brown adipocytes differentiated in culture. J Biol Chem 265:16464-16471

Shepherd JT (1990) Increased systemic vascular resistance and primary hypertension: the expanding complexity. J Hypertens 8:S15-S27

Shixian Q, VanCrey B, Shi J, Kakuda Y, Jiang Y (2006) Green tea extract thermogenesis-induced weight loss by epigallocatechin gallate inhibition of catechol-O-methyltransferase. J Med Food 9:451-458

Takikawa M, Inoue S, Horio F, Tsuda T (2010) Dietary anthocyanin-rich bilberry extract ameliorates hyperglycemia and insulin sensitivity via activation of AMP-activated protein kinase in diabetic mice. J Nutr 140:527-533

Tanida M, Yamamoto N (2011) Central AMP-activated protein kinase affects sympathetic nerve activity in rats. Neurosci Lett 503:167-170

Viollet B, Andreelli F, Jørgensen SB, Perrin C, Flamez D, Mu J, Wojtaszewski JF, Schuit FC, Birnbaum M, Richter E, Burcelin R, Vaulont S (2003) Physiological role of AMP-activated protein kinase (AMPK): insights from knockout mouse models. Biochem Soc Trans 31:216-219

Zapolska-Downar D, Bryk D, Małecki M, Hajdukiewicz K, Sitkiewicz D (2012) Aronia melanocarpa fruit extract exhibits anti-inflammatory activity in human aortic endothelial cells. Eur J Nutr 51:563-572

Zhao ZW, Cai W, Lin YL, Lin QF, Jiang Q, Lin Z, Chen LL (2011) Ameliorative effect of astaxanthin on endothelial dysfunction in streptozotocin-induced diabetes in male rats. Arzneimittelforschung 61:239-246

doi:10.1186/2193-1801-2-626

Cite this article as: Sonoda et al: Anthocyanin-rich Aronia melanocarpa extract improves body temperature maintenance in healthy women with a cold constitution. SpringerPlus 2013 2:626. 